\title{
Research on Novel Pattern and Approach for Mobile Internet Marketing based on Data Mining and Information Fusion Techniques
}

\author{
Shaozong Chai ${ }^{1}$ \\ ${ }^{1}$ Beijing Inistitute of Economics and Management \\ Beijing, 102602 China
}

\begin{abstract}
In this paper, we conduct theoretical research on the novel pattern and approach for mobile Internet marketing based on data mining and information fusion algorithms. At present, with the globalization of economy, Internet technology and information technology as the characteristics of the new economy, the enterprise management put forward higher requirements. In recent years, with the progress of the popularization of computer and network technology, network marketing has been booming. Compared with the traditional marketing, network marketing has the transmission speed, wide range, vivid, rapid feedback, low cost, many advantages. Our method performs well and obtains acceptable result. We will research more on the topic in the future study.
\end{abstract}

Keywords: Data Mining; Mobile Internet Marketing; Information Fusion; Numerical Simulation.

\section{INTRODUCTION}

Internet marketing has changed the way people of the traditional shopping, it is the traditional marketing mode innovation, has special superiority and irreplaceability. However, due to the low level of China's network marketing development overall, network of enormous advantage and potential of marketing has not yet been uncovered. Therefore, the development of the network marketing needs government, enterprises and consumers concerted effort to improve the network consumption environment, update the network consumption idea and actively create conditions to promote the benign development of the network marketing. At present, with the globalization of economy, Internet technology and information technology as the characteristics of the new economy, the enterprise management put forward higher requirements. In recent years, with the progress of the popularization of computer and network technology, network marketing has been booming. The network marketing, refers to the enterprise through the Internet, release the information of goods or services, to accept online orders from customers, and then by mail or by the enterprise door-to-door delivery of a marketing model. Compared with the traditional marketing, network marketing has the transmission speed, wide range, vivid, rapid feedback, low cost, many advantages. Network marketing is a main form of e-commerce, its development momentum is swift and violent with its significant advantage strongly impact the existing economic pattern and the traditional marketing concept. Although network marketing in our country got fast development, but compared with some developed countries, the network marketing of our country starts relatively late, still in the initial growth. At present, in the field of network marketing in China still exist many problems and restricts the further development of network marketing in our country [1-2].

Big data in the Internet of things and cloud computing internal indispensable key elements at the same time also influence every aspect of social life. For marketing and advertising, big data plays an important role, on the one hand, deconstructs the traditional system, at the same 
time, it again to reconstruct a new possibility [3]. We can say the use of the data throughout the entire process of marketing and the marketing effect plays an important role. This article based on the background of big data will focus on marketing system how to deconstruct and reconstruct the internal logic and develop trend. Because of carrying the data information, so during the process of application, the data is no longer merely to record the objective phenomenon or numerous and disorderly, but with a special significance and value. If online analysis is a kind of perspective to data detection, the main purpose of the data mining is found hidden in the data under the surface of the history of law and to make predictions about the future [4]. After entering the 21st century, data visualization become another result of data mining, through the complex data into intuitive graphics, and presented to the most common users, making it easy to understand, the people all of the available tools and instruments. In the following figure one, we should the traditional Internet marketing system structure. On the basis of continuous development and evolution, cloud computing and big data. Big data refers to the size is beyond the traditional sense of the feet therefore, combing machine learning and pattern recognition algorithms into the system is a trend and necessary step.

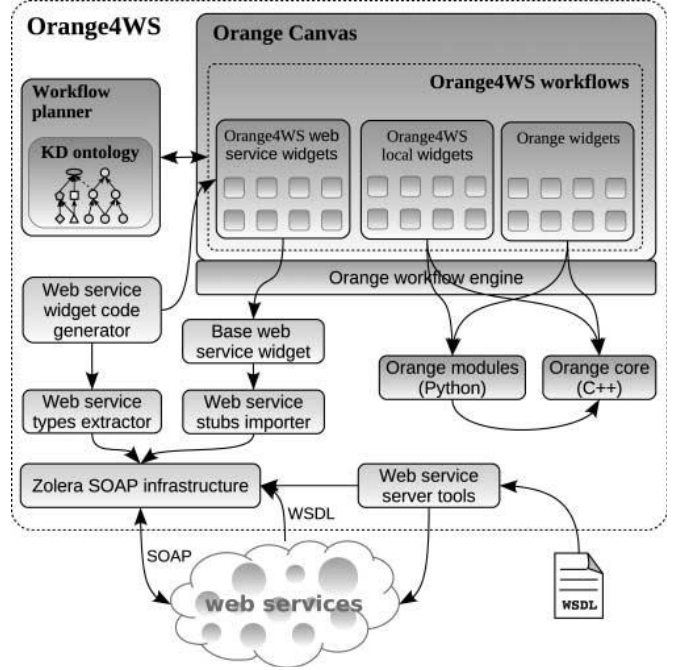

Fig. 1The Sample Structure of Traditional Internet Marketing System
In this paper, we combine the advantage of data mining and information fusion technique to conduct theoretical research on the novel pattern and approach for mobile Internet marketing. In the following sections, we will illustrate our methodology in detail.

\section{The Core Components of Our Proposed Method}

The Data Mining and Clustering Algorithms. In recent years, data mining has attracted a great attention in information industry, its main reason is that there are large amounts of data, can be widely used, and the urgent need to transform these data into useful information and knowledge. Access to information and knowledge can be widely used in various applications, including business management, production control, market analysis, engineering design and scientific exploration, etc. Data mining is extracted from large amounts of data or "mining" knowledge. The term is actually a bit of a misnomer. Note that the gold from ore or sand mining called gold mining, instead of sand and gravel mining. In this way, data mining should be more correctly named "knowledge" in the data mining, unfortunately, it's a bit long. "Knowledge mining" is a short term, may not be able to emphasis on mining from large amounts of data. Mining is, after all, a very vivid terms, it seized from a lot of, raw materials found in small amounts of the characteristics of the process of gold bullion. Data mining step can interact with the user or the knowledge base. Interesting patterns to provide to the user or as a new knowledge stored in knowledge base. Note, according to this view, the step in the process of data mining is the whole, although is the most important step, because it found hidden pattern. The formula one shows the objective function for data mining.

$$
L(X, Y)=\left(\sum_{i=1}^{l}\left|x_{i}-y_{i}\right|^{p}\right)^{1 / p}
$$


A relational database system is widely used in business applications. With the development of database technology, all kinds of advanced database system have appeared and in development, to adapt to the new database application needs. The model by providing rich of dealing with the complex object data types and object orientation, extend the relational model. In addition, it also contains a relational query language of the special structure, so that the management to increase the data type. By increasing the processing complex data types, classes, object inheritance hierarchy and as mentioned above, the object model expands the basic relational model. Object-relational database and application are becoming more popular in the industry. The following expression defines this.

$$
\text { Display - as < Result - from > }
$$

This step is to identify a collection of properties and dimensions, choose the correlation of measurement for them. Due to the different levels of $\mathrm{d}$ for a given class has a different correlation, in principle, define $\mathrm{d}$ concept level of each attribute should be included in the correlation analysis. By removing or generalization has the property of a large number of different values. In other words, if meet the layer transfer threshold, then the method allows the test does not meet the minimum support threshold of children. Each concept layer can have its own threshold. Normally, for a given layer, layer transfer threshold is set to the next layer of minimum support threshold and the minimum support threshold value of a given layer between a value and the user can choose "fall" in a higher level or lower layer transfer threshold, make the child be reviewed at the lower levels the offspring of the frequent items. Reduce the transmission threshold to the lowest layer of minimum support threshold will make all of the offspring be reviewed. The structure is shown below.

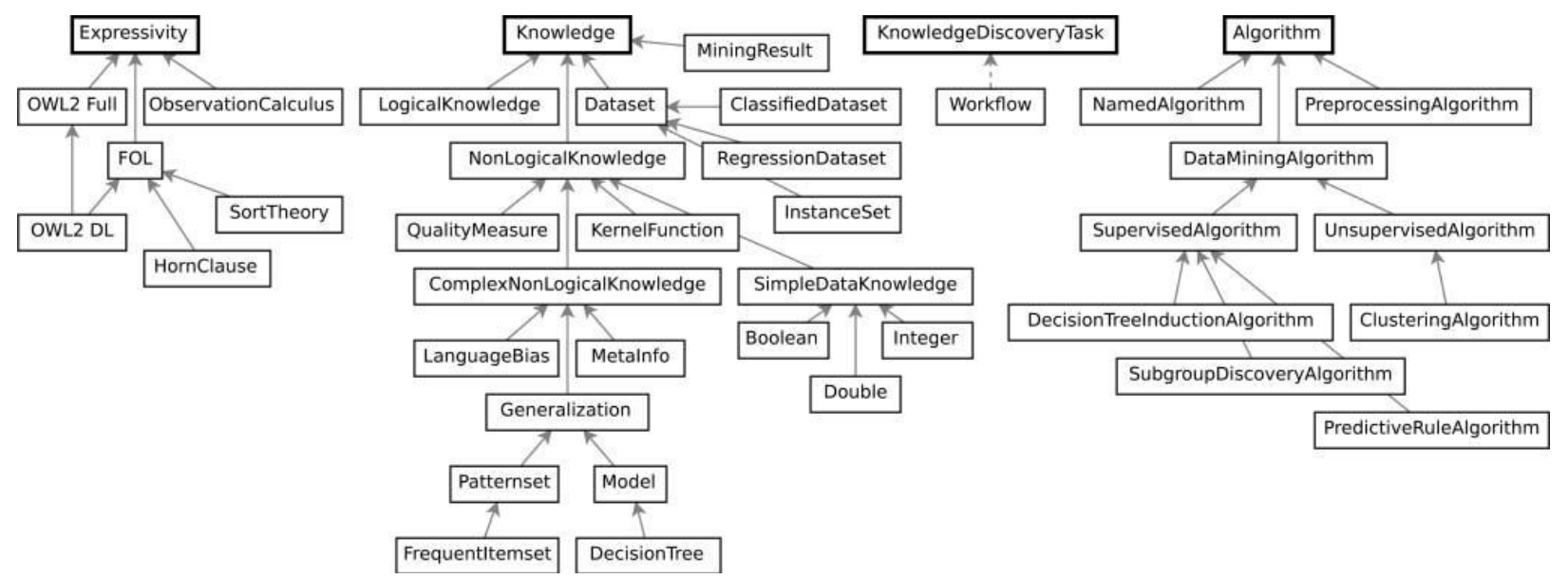

Fig. 2The Structure and Organization of Data Mining Algorithms

Multimedia database for image, audio and video data them to extract based on graph content, voice transmission, video on demand, the world-wide-web spoken and identification based on voice command user interface, etc. Multimedia database must support large objects, because like video data object may need to be billions of bytes of storage. Also need special storage and retrieval technology, because the video and audio data need to be stable, predetermined rate real-time retrieval, prevent the image or voice interrupted and buffer overflow system. This data is called continuous media data.

Concepts of Information Fusion. Heterogeneous database consists of a set of interconnected, autonomy of member database. These members of mutual communication in 
order to exchange information and answer queries and a member of the objects in the database with other members in the database object is very different, making it difficult to their semantic absorbed into an integral part of the heterogeneous database. Although web page looks nice and informative, but they actually are structured and lack of predefined patterns, type and format. In this way, information extraction and data mining for systematically, computer is hard to understand the semantic web and put them in the form of organized structured. Based on keyword search services, and do not understand the specific context of a web page, can provide users with only limited help. For example, a single keyword based web search might return hundreds of pointer, refers to the page containing the keyword, and most of them have nothing to do with users expect to find. Data mining function is used to specify the type to find patterns in a data mining tasks. Generally, data mining tasks can be divided into two categories: description and prediction. Descriptive mining tasks characterize general characteristics of data in the database. In some cases, the user does not know what types of patterns in their data is interesting, so may want to parallel search a variety of different models. In this way, it is important that the data mining system to be able to dig a variety of types of patterns, to adapt to the needs of different users or different applications. In addition, the data mining system should be able to find patterns of various kinds of particle size. The figure three shows the sample structure.

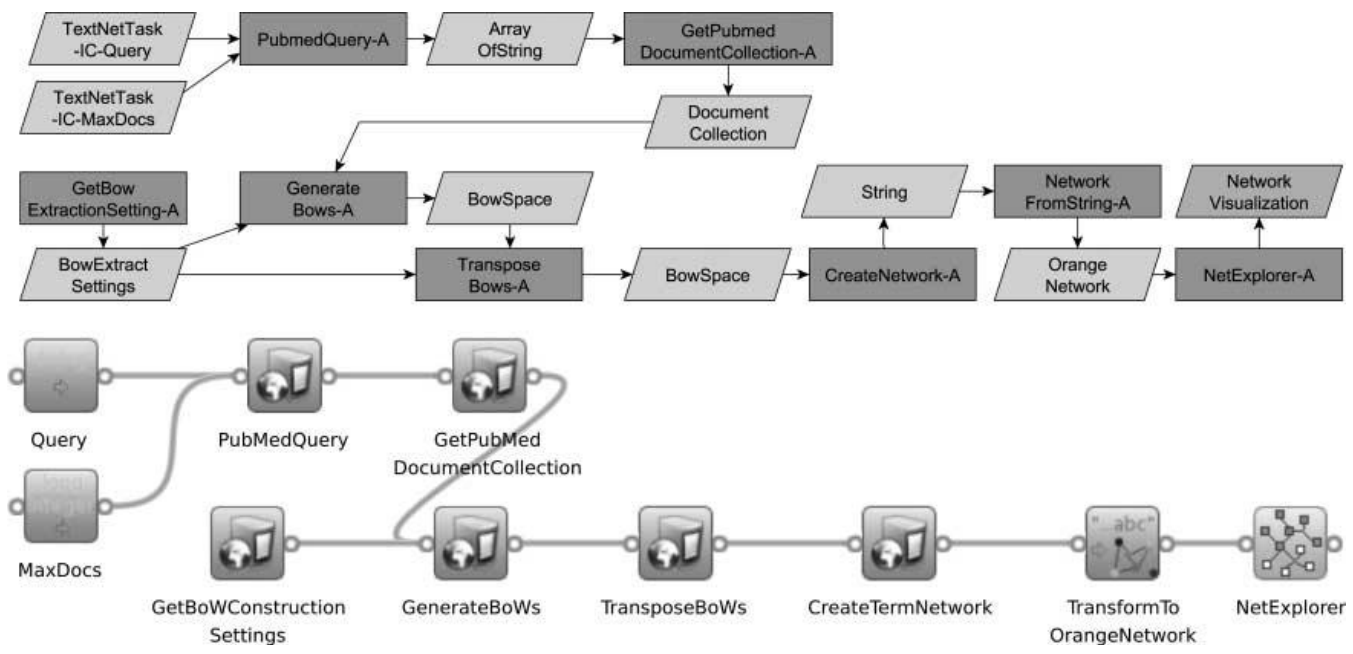

Fig. 3The Sample of the Information Fusion based Marketing Pattern

The Modified Mobile Internet Marketing. As mentioned research, data mining and information technique will enhance the performance of the marketing result. Network marketing is a virtual market, it is the premise of supply and demand both sides trust each other. At present, China's population overall knowledge level is not high, lead to insufficient understanding of network marketing, consumers lack of consumer confidence. Intuitive is poor due to network performance products, consumers worried about the risk of online shopping, such as after-sales service guarantee factors, resulting in market size. Guide the broad masses of consumers to change the past seeing is believing the traditional way of shopping and shopping habits, make its psychologically accept network marketing. Carrying out the academic study of network marketing, and constantly develop new way suitable for the situation of our country's network marketing and the new strategy; Improve the national quality, a large number of network marketing talents. Network marketing is not only need knowledge and marketing skills comprehensive talents, also need the high quality of the consumer. Which of the two aspects of the 
job is not an enterprise or individual can do, only rely on the power of the state to achieve. We believe we will gain better result if use the information based approach.

\section{CONCLUSIONS}

In this paper, we conduct theoretical research on the novel pattern and approach for mobile Internet marketing based on data mining and information fusion algorithms. In recent years, with the progress of the popularization of computer and network technology, network marketing has been booming. The network marketing, refers to the enterprise through the Internet, release the information of goods or services, to accept online orders from customers. Data clustering method could help the companies to throw the advertisement more specifically. In the future research, we plan to combine more information techniques to modify the current research pattern.

\section{References}

[1] Perera, C., Jayaraman, P. P., Zaslavsky, A., Christen, P., \& Georgakopoulos, D. (2014). MOSDEN: An Internet of Things Middleware for Resource Constrained Mobile Devices. Hawaii International Conference on System Sciences (pp.1053-1062). IEEE Computer Society.

[2] Kazakevitch, G., Torlina, L., Hendricks, S., Kazakevitch, G., \& Hendricks, S. (2014). Consumer loyalty versus propensity to switch between providers in mature it marketsPACIS 2006 Proceedings.

[3] Kang H, Kang M, Yoon S, et al. A consumer value analysis of mobile internet protocol television based on a means-end chain theory[J]. Service Business, 2014, 8(4):587-613.

[4] Purwanegara M, Apriningsih A, Andika F. Snapshot on Indonesia Regulation in Mobile Internet Banking Users Attitudes is [J]. Procedia - Social and Behavioral Sciences, $\quad 2014: 147 \quad-\quad 155$. 